\title{
Clinical application of compound Glycyrrhizin tablets in the treatment of patients with Simplex Henoch-Schonlein Purpura and its effect on immune function
}

\author{
Chao $\mathrm{Li}^{1}$, Zhi-bin Wang ${ }^{2}$
}

\begin{abstract}
Objectives: To investigate the curative effect of Compound Glycyrrhizin Tablets in the adjuvant treatment of simplex Henoch-Schonlein purpura and its influence in improving immune function.

Methods: In this retrospective study design was used in this study. Eighty newly diagnosed patients with purpura simplex who visited the outpatient department of Baoding First Central Hospital from June 2017 to February 2020 were included. They were randomly divided into treatment group and control group. The two groups were provided with the same conventional comprehensive treatment. Patients in the treatment group received oral administration of Compound Glycyrrhizin Tablets on the basis of conventional treatment. The clinical efficacy of the treatment group and the control group were compared according to the time and effect of purpura regression, followed by the comparison of changes of T cell subsets before and after treatment.

Results: The total effective rate of the treatment group was $92.5 \%$, which was higher than that of the control group (77.5\%) $(\mathrm{P}<0.05)$. The purpura subsidence time of effective patients in treatment group was shorter than that in control group $(P<0.05)$. There was no significant difference in lymphocyte subsets between the treatment group and the control group before treatment. After treatment, the proportion of CD4+ cells and CD4+/CD8+ cells in the treatment group were obviously higher than that in the control group, and the count of CD8+ cells was evidently lower than that in the control group $(P<0.05)$.

Conclusions: Compound Glycyrrhizin is effective in the adjuvant treatment of simplex Henoch-Schonlein purpura without obvious adverse reactions, which is valuable for clinical application as an adjuvant.
\end{abstract}

KEYWORDS: Compound Glycyrrhizin, Henoch-Schonlein purpura, Lymphocyte subsets.

doi: https://doi.org/10.12669/pjms.38.1.4609

How to cite this:

Li C, Wang Z. Clinical application of compound Glycyrrhizin tablets in the treatment of patients with Simplex Henoch-Schonlein Purpura and its effect on immune function. Pak J Med Sci. 2022;38(1):271-275. doi: https://doi.org/10.12669/pjms.38.1.4609

This is an Open Access article distributed under the terms of the Creative Commons Attribution License (http://creativecommons.org/licenses/by/3.0), which permits unrestricted use, distribution, and reproduction in any medium, provided the original work is properly cited.

1. Chao Li,

Department of Hematology,

Beijing Aerospace General Hospital,

Beijing, 100076, P.R. China.

2. Zhi-bin Wang ${ }^{2}$

Department of Hematology,

Baoding First Central Hospital,

Baoding, Hebei, 071000, P.R.China.

Note: Chao Li and Zhi-bin Wang both considered as co-first authors.

Correspondence:

Chao Li,

Department of Hematology,

Beijing Aerospace General Hospital,

Beijing, 100076, P.R. China.

E-mail: Iclw712@126.com

* Received for Publication:

* Revision Received:

* Revision Accepted:
April 22, 2021

September 8, 2021
August 27, 2021

\section{INTRODUCTION}

Henoch-Schonlein purpura (HSP) is one of the common allergic diseases with extensive small-vessel vasculitis as its basic pathology. Its primary manifestations involve characteristic skin rash, accompanied frequently by gastrointestinal bleeding, renal impairment, abdominal pain, joint pain, etc. Furthermore, there is no consensus yet in the etiology of HSP, which can be induced by the occurrence of allergic reactions to infection, food, drugs, environment and other multiple factors. It can occur at any age, mostly in adolescents, and its incidence peaks in spring and autumn..$^{1-3}$ So far, there is an absence of complete clarification of 
its pathogenesis. According to previous research, immune dysfunction plays an important role in the occurrence and development of diseases. ${ }^{4}$ In addition, there is currently no effective specific method for the treatment of HSP, among which hormone therapy is the major choice, yet with obvious side effects. ${ }^{5}$ Compound Glycyrrhizin Tablet is a compound preparation composed of glycyrrhizin, monoammonium glycyrrhizinate, glycine and methionine. It is a plant extract of glycosides, with the function of immunoregulation. It can treat inflammatory skin diseases associated with T cells such as psoriasis, leukoderma and lichen planus by regulating T cell activation. ${ }^{6}$ Accordingly, in this study, Compound Glycyrrhizin Tablets were used for the adjuvant treatment of simplex HSP, with the purpose to observe its therapeutic effect and impact on the change of lymphocyte subsets.

\section{METHODS}

General Data: The subjects of the study were 80 patients with simplex HSP who were admitted to the Outpatient Department of Hematology in Boding First Central Hospital from June 2017 to February 2020. All patients were included due to the first attack of this disease, and met the relevant diagnostic criteria of HSP. ${ }^{7}$ Of the enrolled 80 cases, there were 43 males and 37 females, with an average age of $15.3 \pm 1.2$ years old ( $5 \sim 36$ years old). The average time from onset to the first outpatient visit was $5.7 \pm 1.5 \mathrm{~d}$ days $(3 \sim 10 \mathrm{~d})$. All patients were randomly divided into the treatment group and the control group, with 40 cases in each group. Among them, there were 21 males and 19 females in the treatment group, with an average age of $15.7 \pm 1.9$ years old (range, 5-36 years old), and the time from onset to the first outpatient visit of $5.9 \pm 1.2 \mathrm{~d}$ days (range, 3 10d). In addition, 22 males and 18 females were grouped in the control group, with an average age of 15.1 \pm 1.7 years old (range, 5-36 years old), and the time from onset to the first outpatient visit of5.4 $\pm 1.4 \mathrm{~d}$ days (range, $3 \sim 10 \mathrm{~d}$ ). There was no significant difference in general data such as gender, age and course of disease between the two groups $(\mathrm{P}>0.05)$.

Ethical approval: The study was approved by the Institutional Ethics Committee of Baoding First Central Hospital on September 10, 2019 (No.: 2012-KY-027), and written informed consent was obtained from all participants

\section{Inclusion Criteria:}

1. Patients who met the diagnostic criteria of HSP.
2. Patients who had no fever, abdominal pain, joint pain and other symptoms except skin purpura.

3. Patients who had no abnormality in routine blood test, routine urine test, routine stool test, liver and kidney function test, Antinuclear Antibodies profile examination, etc.

All patients were required to stay in bed at home, keep a light diet, avoid contact with all suspicious allergens, or hot and cold stimulation, and receive oral medication treatment in the Outpatient Department. Patients in the control group were provided with oral administration of Loratadine, Calcium Preparation, Vitamin C, Compound Rutin and other conventional treatment. Meanwhile, The treatment group was treated with Compound Glycyrrhizin Tablets on the basis of conventional treatment (Manufacturer: Eisai [China] Pharmaceutical Co., Ltd.). As for the therapeutic dosage, it was $25 \mathrm{mg}$ three times a day for patients aged $\leq 12$ years old, while $50 \mathrm{mg}$ three times a day, for those aged $>12$ years old.

Detection Methods: In the treatment group and the control group, $4 \mathrm{ml}$ of fasting blood was collected from each patient in the morning and placed into EDTA anticoagulant tubes for examination. According to the requirement, $10 \mathrm{ul}$ monoclonal antibodies (CD45-FITC/CD4-PE/CD8-ECD/CD3PC5) were added to the numbered tubes. After that, $100 \mathrm{ul}$ of anticoagulant were supplemented into the tube, which was then mixed well, stored in dark, and incubated at room temperature for 15-20 min. In the next step, 500ul Beckmann hemolysin OptiLyse $\mathrm{C}$ was added and reacted for 10 seconds through vortex mixing. After placement in dark for $10-15$ minutes, $300 u$ of $0.9 \%$ sodium chloride was added, shaken and mixed well for detection.

Criteria for Determining Curative Effect Markedly effective: All relevant symptoms disappeared after treatment, with no abnormality in related examinations. Effective: Patients showed obvious improvement in the condition after treatment, yet with no complete recovery. Ineffective: There was no improvement in the condition after treatment, or accompanied by the appearance of new symptoms, signs and auxiliary examination abnormalities. Total effective rate $=$ (Cases of markedly effective+Cases of effective)/Total cases $\times 100 \%$.

Observational Indexes: The two groups of patients were required to have a weekly outpatient review: To observe the regression of skin purpura and record the regression time; to receive routine urine and stool test every week and check the blood biochemistry every two weeks; to observe adverse 
Treatment of Patients with Simplex Henoch-Schonlein Purpura

Table-I: Comparison of clinical curative effect between treatment group and control group [n (\%)].

\begin{tabular}{lcccc}
\hline Groups & Markedly effective & Effective & Ineffective & Total effective rate (\%) \\
\hline Treatment group $(\mathrm{n}=40)$ & $33(82.5)$ & $4(10.0)$ & $3(7.5)$ & $92.5^{*}$ \\
Control group $(\mathrm{n}=40)$ & $26(65.0)$ & $5(12.5)$ & $9(22.5)$ & 77.5 \\
\hline
\end{tabular}

Compared with the control group, ${ }^{*} \mathrm{P}<0.05$.

drug reactions; to evaluate the curative effect after one month; and to measure $\mathrm{T}$ lymphocyte subsets (CD3+, CD4+, CD8+, CD4+/CD8+) by flow cytometry based on the collection of fasting venous blood at admission and the second day after the course of treatment.

Statistical Analysis: Statistical software SPSS 21.0 was used for data analysis in this study. The measurement data were expressed as mean \pm standard deviation $(\mathrm{x} \pm \mathrm{s})$, and compared by using $\mathrm{t}$ test. Meanwhile, the counting data were expressed as percentage or rate, and Chi-square test was used for comparison between groups. $\mathrm{P}<0.05$ meant that the difference was statistically significant.

\section{RESULTS}

The total effective rate was $92.5 \%$ and $77.7 \%$ in the treatment group and the control group, respectively is shown in Table-I. The total effective rate of the treatment group was significantly higher than that of the control group, with statistically significant difference $(\mathrm{P}<0.05)$. The purpura regression time was significantly shorter in the treatment group than that in the control group, and the difference was statistically significant $(\mathrm{P}<0.05$; Table-II).

Adverse reactions: There were no cases of adverse reactions related to the use of Compound Glycyrrhizin Tablets in the treatment group. The results of 1-month follow-up revealed that the three patinets had mno improvement howed the expansion of purpura in the treatment group. While in the
Table-II: Comparison of purpura regression time in effective patients between two groups $(x \pm s, d)$.

\begin{tabular}{lc}
\hline Groups & Purpura subsidence time \\
\hline Treatment group $(\mathrm{n}=37)$ & $5.3 \pm 1.6^{*}$ \\
Control group $(\mathrm{n}=31)$ & $7.9 \pm 1.3$ \\
\hline
\end{tabular}

Compared with the control group, ${ }^{*} \mathrm{P}<0.05$.

control group, 9 cases were ineffective, four cases showed that the skin purpura was enlarged, two cases had abdominal pain repeatedly, and three patients showed positive urine protein by routine urine test. All cases with ineffective outcome were admitted to the hospital for further treatment.

Before treatment, there was no significant difference in the count of lymphocyte subsets between the treatment group and the control group $(\mathrm{P}>$ $0.05)$. While after treatment, the proportion of CD4+ cells and CD4+/CD8+ cells was remarkably higher, while the proportion of CD8+ cells was significantly lower in the treatment group than those in the control group, with statistically significant differences $(\mathrm{P}<0.05)$. In addition, there was no significant difference in $\mathrm{CD} 3+$ cells between the two groups after treatment $(\mathrm{P}>0.05)$. Corresponding results are shown in Table-III.

\section{DISCUSSION}

HSP is a disease with vascular allergy as its pathological basis. It has been documented that

Table-III: Comparison of serum T lymphocyte subsets between the two groups before and after treatment ( $\mathrm{x} \pm \mathrm{s})$.

\begin{tabular}{llcccc}
\hline Time & Groups & CD3+ (\%) & CD4+ $(\%)$ & $C D 8+(\%)$ & $C D 4+/ C D 8+$ \\
\hline \multirow{3}{*}{ Before treatment } & Treatment group $(\mathrm{n}=40)$ & $58.71 \pm 4.32$ & $31.39 \pm 5.26$ & $42.02 \pm 8.53$ & $0.74 \pm 0.21$ \\
& Control group $(\mathrm{n}=40)$ & $58.13 \pm 4.49$ & $31.71 \pm 5.28$ & $42.13 \pm 9.16$ & $0.73 \pm 0.23$ \\
& P value & 0.883 & 0.935 & 0.986 & 0.957 \\
& Treatment group $(\mathrm{n}=37)$ & $72.71 \pm 6.32$ & $44.71 \pm 3.28$ & $30.43 \pm 4.14$ & $1.49 \pm 0.42$ \\
After treatment & Control group $(\mathrm{n}=31)$ & $68.13 \pm 5.49$ & $37.76 \pm 3.71$ & $34.43 \pm 4.63$ & $1.21 \pm 0.49$ \\
& P value & 0.494 & 0.02 & 0.013 & 0.015 \\
\hline
\end{tabular}

Pak J Med Sci January - February 2022 Vol. 38 No. $1 \quad$ www.pjms.org.pk 273 
immune factors are involved significantly in the occurrence and development of the disease. ${ }^{8}$ It is generally considered that the normal immune response of human body depends on various immune cells, among which $T$ cells occupy the most critical position in the process of active immunity. ${ }^{9}$ $\mathrm{T}$ cells interact with or restrict each other to form an appropriate immune response. Meanwhile, T lymphocytes can be divided into different subsets according to their various composition and biological functions. CD3+ is the surface marker of all $\mathrm{T}$ lymphocytes. To be specific, the proposed subsets can be sub-classified into helper/inducible $\mathrm{T}$ lymphocytes (Th) and suppressor/killer $\mathrm{T}$ lymphocytes (Ts), namely, CD4+T cells and CD8+ $\mathrm{T}$ cells. In terms of corresponding functions, CD4+T cells can promote $B$ cells to secrete antibodies and regulate the activation of other $\mathrm{T}$ cells. ${ }^{10-12}$ Simultaneously, CD8+T are identified to be the primary cytotoxic effector cells, which act directly on target cells and exert an inhibitory role in immune function. Under normal circumstances, a stable and balanced immune cell network can be formed on the basis of interaction and restriction between CD4+ $\mathrm{T}$ cells and CD8+ T cells, which can be involved in regulating normal immune function and maintaining immune homeostasis. However, the imbalance between them may result in immune dysfunction in vivo and induce the occurrence of diseases consequently. ${ }^{13}$ The onset of HSP was associated with an imbalance of Th1/Th2 and Th2 preponderance activation, leading to increased secretion of Th2 cytokines. Then, the immune active cells are stimulated to produce inflammatory factors, resulting in structural changes of vascular endothelial cells. After vascular endothelial cell injury, neutrophils are activated to produce a large number of reactive oxygen species (ROS), which further accelerates the progression of HSP. ${ }^{14}$

Concerning the treatment of HSP, there is still no specific drug for the treatment so far, and conventional treatment is the major choice, including anti-allergy, improving vascular permeability, symptomatic therapy, etc. However, patients may have different responses to treatment. ${ }^{15}$ Prior research evidence has documented that Compound Glycyrrhizin has anti-inflammatory, anti-allergic, antiviral and immunomodulatory effects. ${ }^{16}$ Its pharmacological action is generally exerted through the following approaches. Specifically and firstly, it can directly bind to phospholipase A2, the promoter of arachidonic acid metabolism pathway, which can further hinder its phosphorylation selectively and thus inhibit its activation. Significantly, the blockage of arachidonic acid metabolism at the initial stage can restrict the release of prostaglandins, leukotrienes and other inflammatory mediators. Besides, it can also suppress the activation of complement by inhibiting the $\mathrm{C} 2$ level in the classical complement pathway, hence interfering with the release of leukocytes and playing a dual role in preventing inflammation and allergy. Secondly, by inhibiting the destruction of adrenal corticosteroids, Compound Glycyrrhizin can retard the metabolism of glucocorticoids to enhance the physiological effect of glucocorticoids, thereby alleviating inflammatory exudation and small vessel inflammation. In such a way, it exhibits a hormone-like effect and can also avoid hormonelike adverse reactions importantly. Thirdly, it can inhibit the synthesis of immunoglobulin directly or indirectly by altering the distribution of lymphocyte subsets. Consequently, the process may reduce the production of immune complexes, thus suppressing immune response. ${ }^{17-20}$ Fourthly, Compound Glycyrrhizin also has a role in weakening the inflammatory reaction by reducing oxygen radicals in vivo to alleviate the damage of superoxide to blood vessels. In recent decades, the application of Compound Glycyrrhizin Tablets has achieved a relatively satisfactory outcome in the treatment of urticaria, psoriasis, alopecia areata and various other immune related diseases. ${ }^{21-22}$

In our study, outpatient treatment using Compound Glycyrrhizin Tablets obtained satisfactory clinical results in the adjuvant treatment of simplex HSP. The total effective rate was $92.5 \%$ in the treatment group, and the purpura regression time was $5.3 \pm 1.6$ days in effective patients. Corresponding difference was statistically significant when compared with the control group. Previous studies have also shown that the treatment regimen of compound glycyrrhizin tablets combined with glucocorticoids can significantly reduce the levels of febrile inflammatory factors in peripheral blood of patients with HSP without renal injury. ${ }^{23}$ At the same time, 3 ineffective patients in the treatment group had no renal damage, while three of nine had no improvement in the control group had positive urine protein. Of course, owing to a limited sample size of the present study, it still remains unclear whether it is suggested that Compound Glycyrrhizin can reduce the incidence of renal damage in HSP. There is a need to further observe the curative effect based on a larger sample size and expanded scope of research. In addition, 
as indicated in our study, the treatment group was superior to the control group in improving serum $\mathrm{T}$ lymphocyte subsets. Following the use of Compound Glycyrrhizin, those patients with simplex HSP had obviously higher count of CD4+T cells while much lower count of CD8+T than those with conventional treatment in the control group. Collectively, findings in our study support that Compound Glycyrrhizin can improve the immune function of patients with simplex HSP.

Limitations of this study: The number of subjects included in this study is limited, so the conclusions drawn may not be very convincing. In addition, the subjects of this study are outpatients, so there may be some non-response bias in the follow-up, which may have some influence on the results of the study.

\section{CONCLUSIONS}

Outpatient use of Compound Glycyrrhizin Tablets has an improved curative effect in the treatment of patients with simplex HSP. It can accelerate purpura regression, shorten the course of treatment, reduce the hospitalization rate, and have no obvious adverse drug reactions. Therefore, Compound Glycyrrhizin Tablets is useful for clinical application as an adjuvant therapy for patients with HSP.

\section{Conflicts of interest: None.}

Source of funding: None.

\section{REFERENCES}

1. Hetland LE, Susrud KS, Lindahl KH, Bygum A. Henoch-Schönlein Purpura: A Literature Review. Acta Derm Venereol. 2017;97(10):11601166. doi: 10.2340/00015555-2733

2. Roache-Robinson P, Hotwagner DT. Henoch Schönlein Purpura. 2020 Dec 2. In: StatPearls [Internet]. Treasure Island (FL): StatPearls Publishing; 2021 Jan-.

3. Di Pietro GM, Castellazzi ML, Mastrangelo A, Montini G, Marchisio P, Tagliabue C. Henoch-Schönlein Purpura in children: not only kidney but also lung. Pediatr Rheumatol Online J. 2019;17(1):75. doi: 10.1186/s12969-019-0381-y

4. Heineke MH, Ballering AV, Jamin A, Ben Mkaddem S, Monteiro RC, Van Egmond M. New insights in the pathogenesis of immunoglobulin A vasculitis (Henoch-Schönlein purpura). Autoimmun Rev. 2017;16(12):1246-1253. doi:10.1016/j.autrev.2017.10.009.

5. Kawasaki Y, Ono A, Ohara S, Suzuki Y, Suyama K, Suzuki J, et al. Henoch-Schönlein purpura nephritis in childhood: pathogenesis, prognostic factors and treatment. Fukushima J Med Sci. 2013;59(1):1526. doi: $10.5387 / \mathrm{fms} .59 .15$.

6. Yang D, Zheng J, Zhang Y, Jin Y, Gan C, Bai Y. Total glucosides of paeony capsule plus compound glycyrrhizin tablets for the treatment of severe alopecia areata in children: A randomized controlled trial. Evid Based Complement Alternat Med. 2013;2013:378219. doi: $10.1155 / 2013 / 378219$

7. Cui J, Huang LY, Guo J, Wu CR, Zhang B. Diagnosis and treatment of adult mixed-type Henoch-Schönlein purpura. Cent Eur J Immunol. 2019;44(2):138-143. doi: 10.5114/ceji.2019.87064

8. Chen JY, Mao JH. Henoch-Schönlein purpura nephritis in children: incidence, pathogenesis and management. World J Pediatr. 2015;11(1):29-34. doi: 10.1007/s12519-014-0534-5
9. Maciolek JA, Pasternak JA, Wilson HL. Metabolism of activated T lymphocytes. Curr Opin Immunol. 2014;27:60-74. doi: 10.1016/j. coi.2014.01.006

10. Mousset CM, Hobo W, Woestenenk R, Preijers F, Dolstra H, van der Waart AB. Comprehensive Phenotyping of $\mathrm{T}$ Cells Using Flow Cytometry. Cytometry A. 2019;95(6):647-654. doi: 10.1002/ cyto.a.23724

11. Schuh E, Berer K, Mulazzani M, Feil K, Meinl I, Lahm H, et al. Features of Human CD3+CD20+ T Cells. J Immunol. 2016;197(4):1111-7. doi: 10.4049/jimmunol.1600089

12. Brummelman J, Pilipow K, Lugli E. The Single-Cell Phenotypic Identity of Human CD8+ and CD4+ T Cells. Int Rev Cell Mol Biol. 2018;341:63-124. doi: 10.1016/bs.ircmb.2018.05.007

13. Overgaard NH, Jung JW, Steptoe RJ, Wells JW. CD4+/CD8+ doublepositive T cells: more than just a developmental stage? J Leukoc Biol. 2015;97(1):31-38. doi: 10.1189/jlb.1RU0814-382

14. Zhu Y, Dong Y, Wu L, Deng F. Changes of inflammatory mediators and oxidative stress indicators in children with Henoch-Schönlein purpura and clinical effects of hemoperfusion in the treatment of severe Henoch-Schönlein purpura with gastrointestinal involvement in children. BMC Pediatr. 2019;19(1):409. doi: 10.1186/ s12887-019-1802-2

15. Ozen S, Marks SD, Brogan P, Groot N, de Graeff N, Avcin T, et al European consensus-based recommendations for diagnosis and treatment of immunoglobulin A vasculitis-the SHARE initiative. Rheumatology (Oxford). 2019;58(9):1607-1616. doi: 10.1093/ rheumatology/kez041

16. Li QL, He DH, Huang YH, Niu M. Thromboangiitis obliterans in two brothers. Exp Ther Med. 2013;6(2):317-320. doi: 10.3892/ etm. 2013.1160

17. Yan M, Fang PF, Li HD, Xu P, Liu YP, Wang F, et al. Lack of effect of continuous glycyrrhizin administration on the pharmacokinetics of the P-glycoprotein substrate talinolol in healthy volunteers. Eur J Clin Pharmacol. 2013;69(3):515-21. doi: 10.1007/s00228-012-1391-6

18. Vu SH, Bernardo Reyes AW, Ngoc Huy TX, Min W, Lee HJ, Kim $\mathrm{HJ}$, et al. Transcriptomic profiling of phospholipase A2 and the role of arachidonic acid during Brucella abortus 544 infection in both in vitro and in vivo systems. Microb Pathog. 2021;152:104655. doi: 10.1016/j.micpath.2020.104655

19. Xie C, Li X, Wu J, Liang Z, Deng F, Xie W, et al. Anti-inflammatory Activity of Magnesium Isoglycyrrhizinate Through Inhibition of Phospholipase A2/Arachidonic Acid Pathway. Inflammation. 2015;38(4):1639-48. doi: 10.1007/s10753-015-0140-2

20. Ren X, Zhang M, Chen L, Zhang W, Huang Y, Luo H, et al. The antiinflammatory effects of Yunnan Baiyao are involved in regulation of the phospholipase A2/arachidonic acid metabolites pathways in acute inflammation rat model. Mol Med Rep. 2017;16(4):4045-4053. doi: $10.3892 / \mathrm{mmr} .2017 .7104$

21. Liu Q, Jiao Z, Zhong M, Zhang M, Geng F, Zhao H. Effect of long-term coadministration of compound glycyrrhizin tablets on the pharmacokinetics of mycophenolic acid in rats. Xenobiotica. 2016;46(7):627-633. doi: 10.3109/00498254.2015.1103386

22. Lin W, Yu Q, Qin Y, Dai L, Xiao J, Jiao L, et al. To explore the clinical efficacy of Traditional Chinese Medicine bath in the treatment of psoriasis vulgaris with blood-heat syndrome and its effect on related cytokines based on different temperature and different concentration. Medicine (Baltimore). 2020;99(19):e20172. doi: 10.1097/MD.0000000000020172

23. Wang J, Zheng Y, Chen G, Lv Y, Lian F, Jiang D, et al. The changes in pyroptosis-related inflammatory factors in the peripheral blood of patients with Henoch-Schonlein purpura. Ann Palliat Med. 2021;10(6):6687-6693. doi: 10.21037/apm-21-1227.

\section{Authors' Contributions:}

CLi and ZW designed this study and prepared this manuscript, and are responsible and accountable for the accuracy or integrity of the work. CL collected and analyzed clinical data.

ZW significantly revised this manuscript. 